\title{
Are Patients Frequently Readmitted to the Hospital Different from the Other Admitted Patients?
}

\author{
Maribeth Porter, MD, MSCR, David Quillen, MD, Denny Fe Agana, PhD, MPH, \\ Lisa Chacko, MD, MPH, Kimberly Lynch, MSHI, Lauren Bielick, BSN, \\ Xiaoqing Fu, MS, Yang Yang, PhD, and Peter J. Carek, MD, MS
}

Introduction: Although the characteristics of readmitted patients associated with a family medicine inpatient service have been reported, differing characteristics between groups of patients based on readmission rates have not been studied. The aim of this project was to examine patients with differing rates of readmission.

Methods: Patients admitted to a family medicine inpatient service were classified into 1 of 3 groups based on the number of admission and readmissions in a given year. Demographic data and other characteristics of these patients were collected and used in analysis. Descriptive statistics were used to characterize the 3 groups of admissions. Differences in characteristics of groups were compared using Wilcoxon rank sum test for continuous variables and $\chi^{2}$ test or Fisher exact test for categoric variables. Multivariate logistic regressions were used for predicting high-frequency readmission.

Results: Patients in the high-frequency readmission group more commonly had a psychiatric, substance abuse, and chronic pain diagnosis. The primary discharge diagnoses among the 3 groups were similar. Age-group, Charlson severity index, Morse Fall Scale medication list, and problem list were significant for predicting high frequency of readmission. Annually, patients in the high-frequency readmission group had about an $80 \%$ turnover rate.

Conclusions: Although this study examined patient care data from only one large academic health center hospital, the results found that patients who experience 3 or more readmissions in a calendar are associated with specific characteristics. In addition, the list of specific individual patients considered to be high utilizers for hospital readmissions was dynamic and significantly changed during 3 consecutive years. (J Am Board Fam Med 2019;32:58-64.)

Keywords: Hospitalization, Patient Care, Patient Discharge, Patient Readmission

Readmissions following hospital discharge are common and used by many individuals and organizations as a measure of the quality of care provided by physicians and hospitals. ${ }^{1-3}$ As reported by the Centers for Medicare Services, ${ }^{4}$ approximately 1 in 5 patients are readmitted to the hospital within

This article was externally peer reviewed.

Submitted 13 February 2018; revised 12 September 2018; accepted 14 September 2018.

From the Department of Community Health and Family Medicine (MP, DQ, DFA, LC, KL, LB, and PJC) and Department of Biostatistic (XF and YY), University of Florida, Gainesville, FL.

Funding: none.

Conflict of interest: none declared.

Corresponding author: Peter J. Carek, MD, MS, Department of Community Health and Family Medicine, College of Medicine, University of Florida, P.O. Box 100237, Gainesville, FL 32610-0237 (E-mail: carek@ufl.edu).
30 days of discharge. In an attempt to reduce the readmission rates, the Hospital Readmissions Reduction Program was added to the Affordable Care Act. Health care organizations and practitioners are interested in reducing readmission rates, as hospitals in the United States are now financially penalized if their readmission rates for these patients are above a predetermined level. ${ }^{5}$

Numerous attempts to reduce readmission rates for hospitals have been described. Although several single-center studies have demonstrated that a hospital's readmission rate is modifiable, authors of systematic reviews were unable to consistently identify a single intervention or a bundle of interventions that reliably reduced the risk of readmission in a manner that could be considered generalizable. $^{6-10}$ 
Several studies have examined specific patient populations and their risk factors for hospital readmissions. ${ }^{1-16}$ For example, a systematic review of hospital readmissions in elderly patients found that previous hospital admissions (before index admission), longer duration of hospital stay, morbidity/ comorbidity, and functional disability were common risk factors. ${ }^{11}$ Patients readmitted to a family medicine inpatient service had more hospitalizations, more emergency department visits, longer hospital stays, more comorbidities, more discharge medications and higher Charlson scores. ${ }^{13-15}$ Being married had a protective effect for hospital readmission (odds ratio, $0.54 ; 95 \%$ CI, 0.30 to 0.97 ). ${ }^{13}$ Among the Medicaid population, increased number of chronic conditions, medication noncompliance, postdischarge care environments, and substance abuse comorbidities were found to increase the risk of readmission. ${ }^{14} \mathrm{~A}$ broad array of social factors (ie, low socioeconomic status, living situation, lack of social support, marital status, and risk behaviors such as smoking, cocaine use, and medical/visit nonadherence) affect the risk of hospital readmission and mortality in patients with communityacquired pneumonia and heart failure. ${ }^{12}$

Interestingly, a small proportion of patients accounts for a disproportionate number of hospital admissions and health care costs. ${ }^{13}$ Noted over 30 years ago, total hospital billings were concentrated on a few patients (13\%) who consumed as many resources as all other patients combined. ${ }^{17}$ These patients have high rates of substance use, homelessness, social isolation, and lack of a medical home. ${ }^{18-19}$

Although the characteristics of readmitted patients associated with a family medicine inpatient service have been reported, the characteristics between groups of patients based on differing readmission rates have not been studied. ${ }^{13,15}$ The aim of this project was to examine patients with a high and low rate of readmission as well as those patients not readmitted over a 3 -year period.

\section{Methods}

Participants were individuals over the age of 18 years admitted to a family medicine teaching inpatient service at a large academic medical center in the southeast United States between January 1, 2014, and December 31, 2016. Patients were classified into 1 of 3 groups: hospital users without readmission (single admission [SA]), hospital users with low-frequency readmissions (LfR; 1 to 2 readmissions in any given year, not including the index admission), and hospital users with highfrequency readmissions (HfR; 3 or more readmissions in any given year, not including the index admission). A patient was only classified into 1 of the groups. A readmission was defined as an admission occurring within 30 days of a previous hospital admission.

The data used in this study were obtained from the hospital's administrative informational system (Epic Data Warehouse). Demographic data, including sex, age, home zip code, race, ethnicity, marital status, and insurance status, were collected. In addition, length of stay of the index admission; readmissions (including number, time of day, and day of week); length of stay per each admission; primary discharge diagnoses; presence of psychiatric, substance abuse, or chronic pain diagnoses; medication list (number of medications); problem list (number of problems); Charlson Comorbidity Index (CCI); and Morse Fall Scale (MFS) were also obtained. Patients were also grouped by age (young, $<40$ years of age; middle, $\geq 40$ and $<60$ years of age; old, $\geq 60$ years of age), CCI (low, $\leq 1$; medium, 2 to 4 ; and high, $\geq 5$ ), MFS ( 0 to $24=$ no risk, 25 to $50=$ low risk, and $\geq 51=$ high risk), and zip code (east area including 32601, 32609, 32640, and 32604; west area including 32603, 32607, 32608 , and 32656; and other). The number of patient deaths in each readmission group was obtained.

\section{Data Analysis}

Descriptive statistics were used to characterize the 3 groups of subjects. Differences in the characteristics of inpatients were compared between readmission frequency levels by using the Wilcoxon rank sum test for continuous variables and $\chi^{2}$ test or Fisher exact test for categoric variables. Multivariate logistic regressions were used for predicting HfR among all patients. All analyses were performed using $\mathrm{R}$ (version 3.3.0). Significance was defined at a confidence level of $P<.05$.

\section{Results}

A total of 2621 unique patients were admitted during the 3-year study period, with 897 patients being in the LfR group and 314 patients in the HfR 
Table 1. Demographic Data of Patients Admitted and Readmitted to the Hospital

\begin{tabular}{|c|c|c|c|c|c|c|}
\hline \multirow[b]{2}{*}{ Demographic } & \multirow[b]{2}{*}{ SA } & \multirow[b]{2}{*}{ LfR (1 to 2$)$} & \multirow[b]{2}{*}{$\operatorname{HfR}(\geq 3)$} & \multicolumn{3}{|c|}{$P$ value } \\
\hline & & & & SA vs LfR & LfR vs HfR & SA vs HfR \\
\hline Patients & 1410 & 897 & 314 & NA & NA & NA \\
\hline Age, mean (SD) & $58.7(15.9)$ & $56.2(18.2)$ & $56.1(16.0)$ & .0099 & .63 & .019 \\
\hline \multicolumn{7}{|l|}{ Sex } \\
\hline Male, no. (\%) & $555(39.4)$ & $361(40.2)$ & $153(48.7)$ & .70 & .011 & .0028 \\
\hline Female, no. (\%) & $855(60.6)$ & $536(59.8)$ & $161(51.3)$ & & & \\
\hline \multicolumn{7}{|l|}{ Race } \\
\hline White, no. (\%) & $838(59.4)$ & $527(58.8)$ & $175(55.7)$ & .92 & .40 & .33 \\
\hline Black, no. (\%) & $513(36.4)$ & $330(36.8)$ & $128(40.8)$ & & & \\
\hline Other, no. (\%) & $59(4.2)$ & $40(4.5)$ & $11(3.5)$ & & & \\
\hline \multicolumn{7}{|l|}{ Ethnicity } \\
\hline Hispanic, no. (\%) & $41(2.9)$ & $32(3.6)$ & $13(4.1)$ & .45 & 0.77 & 0.34 \\
\hline Non-Hispanic, no. (\%) & $1369(97.1)$ & $865(96.4)$ & $301(95.9)$ & & & \\
\hline \multicolumn{7}{|l|}{ Zip Code } \\
\hline East area & $461(32.7)$ & $277(30.9)$ & $111(35.4)$ & .056 & .34 & .19 \\
\hline West area & $307(21.8)$ & $168(18.7)$ & $54(17.2)$ & & & \\
\hline Other & $642(45.5)$ & $452(50.4)$ & $149(47.5)$ & & & \\
\hline \multicolumn{7}{|l|}{ Marital Status } \\
\hline Single, no. (\%) & $681(48.3)$ & $466(52.0)$ & $183(58.3)$ & .23 & .15 & .0059 \\
\hline Married, no. (\%) & $561(39.8)$ & $332(37.0)$ & $100(31.8)$ & & & \\
\hline Other, no. (\%) & $168(11.9)$ & $99(11)$ & $31(9.9)$ & & & \\
\hline \multicolumn{7}{|l|}{ Insurance Status } \\
\hline Medicare, no. (\%) & $668(47.4)$ & $428(47.7)$ & $167(53.2)$ & $<.001$ & .021 & $<.001$ \\
\hline Medicaid, no. (\%) & $257(18.2)$ & $227(25.3)$ & $87(27.7)$ & & & \\
\hline Other, no. (\%) & $485(34.4)$ & $242(27.0)$ & $60(19.1)$ & & & \\
\hline Deaths, no. (\%) & $156(11.1)$ & $193(21.5)$ & $120(38.2)$ & $<.001$ & $<.001$ & $<.001$ \\
\hline
\end{tabular}

SD, standard deviation; SA, single admissions; LfR, low-frequency readmissions; HfR, high-frequency admissions; NA, not applicable.

group (Table 1). Patients in the SA group were significantly older than the patients in either the LfR or HfR groups. The HfR group had a higher proportion of males and single patients compared with the SA and LfR groups. The SA group had a lower percentage of patients with Medicare or Medicaid compared with the LfR and HfR groups. No differences in the race, ethnicity, or zip code were noted.

The average hospital length of stay was the longest in the HfR group (Table 2). Patients in the HfR group more commonly had a psychiatric, substance abuse, and chronic pain diagnosis compared with the other groups. Furthermore, the HfR group had significantly more medications and problems listed as well as a significantly higher mean CCI and MFS, especially when compared with the SA group.

The primary discharge diagnoses among the 3 groups were similar (Table 3). As noted, 6 of the 10 most frequent diagnoses were common among all groups. Diagnoses related to the cardiopulmonary system were the most common. Chest pain, septicemia, chronic obstructive pulmonary disease, pneumonia, and heart failure were common among all groups.

As noted by the results of the multivariate logistic regression analysis (Table 4), only age group, CCI, MFS, medication list, and problem list were statistically significant for predicting high frequency of readmission. Patients aged 40 years or older had much lower odds of readmission as compared with younger patients. No statistical difference between patients of 40 to 59 years old and those aged 60 years or above was noted. As expected, high levels of CCI scores were found to be associated with much higher odds of readmission. Compared with patients with a CCI of 1 or lower, patients with a CCI between 2 and 4 were nearly 4 times as likely (in terms of odds), and patients with CCI of 5 or above were 9.6 times as likely, to have 3 or more readmissions in any given year. Only 
Table 2. Medical Characteristics of Patients Admitted and Readmitted to the Hospital

\begin{tabular}{|c|c|c|c|c|c|c|}
\hline \multirow[b]{2}{*}{ Characteristic } & \multirow[b]{2}{*}{ SA } & \multirow[b]{2}{*}{ LfR (1 to 2$)$} & \multirow[b]{2}{*}{$\operatorname{HfR}(\geq 3)$} & \multicolumn{3}{|c|}{$P$ value } \\
\hline & & & & SA vs. LfR & LfR vs. HfR & SA vs. HfR \\
\hline Length of stay, mean $( \pm$ SD) & $2.9(2.9)$ & $4.5(2.9)$ & $5.0(3.5)$ & $<.001$ & $<.001$ & $<.001$ \\
\hline \multicolumn{7}{|l|}{ Psychiatric diagnosis* } \\
\hline Yes & $602(42.7)$ & $413(46.0)$ & $174(55.1)$ & .61 & .0063 & $<.001$ \\
\hline \multicolumn{7}{|l|}{ Substance abuse diagnosis* } \\
\hline Yes & $187(13.0)$ & $115(12.7)$ & $56(19.1)$ & .56 & .0071 & .0095 \\
\hline No & $1223(87.0)$ & $782(87.3)$ & $258(80.9)$ & & & \\
\hline \multicolumn{7}{|l|}{ Chronic pain diagnosis* } \\
\hline Yes & $102(6.9)$ & $71(7.8)$ & $37(12.9)$ & .97 & .0089 & .0033 \\
\hline No & $1308(93.1)$ & $826(92.2)$ & $277(87.1)$ & & & \\
\hline Problems list, mean ( $\pm \mathrm{SD})$ & $12.7(8.2)$ & $13.7(9.7)$ & $19.5(10.7)$ & .11 & $<.001$ & $<.001$ \\
\hline Charlson index, mean $( \pm S D)$ & $2.0(2.1)$ & $3.0(2.7)$ & $4.6(2.7)$ & $<.001$ & $<.001$ & $<.001$ \\
\hline Morse Fall Score, mean $( \pm \mathrm{SD})^{*}$ & $48.6(19.0)$ & $50.2(17.5)$ & $52.8(14.8)$ & $<.001$ & .0049 & $<.001$ \\
\hline
\end{tabular}

SD, standard deviation; SA, single admissions; LfR, low-frequency readmissions; HfR, high-frequency admissions.

*Diagnosis found on problem list.

patients with a low risk of fall were significantly associated with a high rate of readmission.

Overall, a total of 314 patients were readmitted to the hospital 3 or more times in a calendar year during the study period (131 patients in 2014, 116 patients in 2015, and 125 patients in 2016). No significant differences were noted in sex, race, ethnicity, zip code, marital status, and insurance status between the 3 cohorts. The 2015 list contained 25 patients $(22.0 \%)$ who are on the same list in 2014, and the 2016 list contains 26 patients (20.8\%) who are on the same list in 2015. Ten patients were on

Table 3. Top Primary Discharge Diagnoses

\begin{tabular}{|c|c|c|c|c|c|}
\hline \multicolumn{2}{|l|}{ SA } & \multicolumn{2}{|l|}{ LfR } & \multicolumn{2}{|l|}{ HfR } \\
\hline Diagnosis & $\mathrm{N}$ & Diagnosis & $\mathrm{N}$ & Diagnosis & $\mathrm{N}$ \\
\hline Chest pain NOS (78650) & 90 & Septicemia NOS (0389) & 38 & $\begin{array}{l}\text { Obs chro bronc w(ac) exac } \\
\quad(49121)\end{array}$ & 16 \\
\hline Chest pain NEC (78659) & 88 & Chest pain NEC (78659) & 27 & Chest pain (NEC) & 12 \\
\hline Septicemia NEC (0389) & 49 & $\begin{array}{l}\text { Obs chro bronc w(ac) exac } \\
\quad(49121)\end{array}$ & 21 & $\begin{array}{l}\text { Pneumonia, organism NOS } \\
\quad(486)\end{array}$ & 9 \\
\hline Syncope and collapse (7802) & 44 & $\begin{array}{l}\text { Acute kidney failure NOS } \\
\text { (5849) }\end{array}$ & 21 & Septicemia NEC (0389) & 8 \\
\hline $\begin{array}{l}\text { Obs chro bronc w(ac) exac } \\
\quad(49121)\end{array}$ & 38 & Acute pancreatitis (5770) & 17 & Chest pain NOS (78650) & 8 \\
\hline $\begin{array}{l}\text { Pneumonia, organism NOS } \\
\quad(486)\end{array}$ & 34 & Chest pain NOS (78650) & 17 & $\begin{array}{l}\text { Urinary tract infection NOS } \\
(5990)\end{array}$ & 7 \\
\hline Atrial fibrillation (42731) & 29 & $\begin{array}{l}\text { Pneumonia, organism NOS } \\
\quad(486)\end{array}$ & 15 & $\begin{array}{l}\text { Hb-SS disease with crisis } \\
\quad(28262)\end{array}$ & 6 \\
\hline $\begin{array}{l}\text { Acute kidney failure NOS } \\
\text { (5849) }\end{array}$ & 29 & $\begin{array}{l}\text { Acute on chronic systolic heart } \\
\text { failure }(42823)\end{array}$ & 12 & $\begin{array}{l}\text { Acute on chronic systolic heart } \\
\text { failure (42823) }\end{array}$ & \\
\hline $\begin{array}{l}\text { Urinary tract infection NOS } \\
(5990)\end{array}$ & 25 & Hypertension NOS (4019) & 11 & $\begin{array}{l}\text { Antineoplastic chemotherapy } \\
\text { encounter (V5811) }\end{array}$ & 6 \\
\hline Cellulitis of leg (6826) & 24 & $\begin{array}{l}\text { Subendocardial infarction, } \\
\text { initial (41071) }\end{array}$ & 10 & $\begin{array}{l}\text { Acute on chronic diastolic heart } \\
\text { failure (42833) }\end{array}$ & 5 \\
\hline
\end{tabular}

SA, single admissions; LfR, low-frequency readmissions; HfR, high-frequency admissions; NOS, not otherwise specified; NEC, not elsewhere classifiable; Hb-SS, sickle cell disease; Obs chro bronc w(ac) exac; obstructive chronic bronchitis with acute exacerbation. 
Table 4. Patient Characteristics at Time of Initial Admission that Predict High-Frequency Readmission

\begin{tabular}{llcr}
\hline & & \multicolumn{1}{c}{ HfR vs. Cohorts SA + LfR } \\
Predictor & \multicolumn{1}{c}{ Category } & Odds Ratio $(95 \%$ CI $)$ & $P$ value \\
\hline Age group & Young $(<40$ years of age $)$ & $2.919(1.921-4.140)$ & $<.001$ \\
& Middle $(\geq 40$ and $<60$ years of age $)$ & $1.582(1.190-2.103)$ & .002 \\
CCI & Old $(\geq 60$ years of age $)$ & - & - \\
& Low $(\leq 1)$ & $1.952(1.476-2.598)$ & $<.001$ \\
Modium $(2-4)$ & $8.815(5.725-13.859)$ & $<.001$ \\
Marital status & High $(\geq 5)$ & - & -024 \\
Medication list & No risk $(0-24)$ & $1.952(1.476-2.598)$ & .022 \\
Problem list & Low risk $(25-50)$ & $1.358(1.042-1.1772)$ & .026 \\
\hline
\end{tabular}

CI, confidential interval; SA, single admissions; LfR, low-frequency readmissions; HfR, high-frequency admissions; CCI, Charlson Comorbidity Index.

the list in 2014, 2015, and 2016 (8\% using the 2016 list as the denominator). For 2014, 2015, and 2016, the mortality rates were $41.2 \%(54 / 131), 37.1 \%$ $(43 / 116)$, and $34.4 \%(43 / 125)$, respectively.

\section{Discussion}

In general, the group of patients experiencing a HfR had several unique characteristics compared with the other patient groups studied (Tables 1 and 2). For instance, the mean age of the patients in both readmission groups was significantly lower than the mean age of all other patients admitted. Although a similar finding was found in patients readmitted for heart failure, acute myocardial infarction, and pneumonia, this finding seems counterintuitive, as older patients are more often frail and would seem to have a higher risk of readmission. ${ }^{20}$ Patients in the HfR group were more likely to be single or divorced compared with the other 2 groups. This finding is consistent with the belief that social support as provided by a marriage or close personal relationship decreases the risk of readmission. Finally, Medicaid serves low-income patients, and socioeconomic status is known to be associated with a higher risk of readmission. ${ }^{20}$ Our data showed higher proportions of patients with Medicaid or Medicare that reaffirms this previously held belief (Table 1).

The HfR group had a higher proportion of patients with either a psychiatric, substance abuse, or chronic pain diagnosis compared with the other groups studied. This finding has also been observed in prior studies, and the presence of similar comorbidities might be an important predictor of readmission risk. In addition, patients in this group had more medications and problems listed in their medical record. Finally, the CCI and MFS are increased in the patients who are readmitted and are highest in the HfR group.

Hospital readmissions have been attributed, at least in some part, to poor or incomplete care in the hospital. We found that discharge diagnoses were similar across the groups. Although one theory is that patients were discharged too soon, another possibility is that some patients may be unable to care for themselves independently. In fact, patients in the HfR group had the longest length of stay compared with the other groups of patients. $\mathrm{Pa}-$ tients in the HfR group also had a number of characteristics that, when combined, might be too challenging for individuals to manage on their own. Hospital systems and/or health care providers may need to create strategies to help some high-risk patient populations manage their disease as opposed to primarily focusing on the disease itself. These strategies could include the use of a registered nurse health coach in the clinic primarily responsible for the patient.

The results of this study may provide insight into the readmission risk for certain patients. For example, physicians may not intuitively be as concerned about discharging a younger patient as opposed to someone older. But our study found that younger patients had a higher risk of readmission, 
so providers might take this into consideration at discharge. Single patients may benefit from additional support provided by a health care provider conducting home visits and other activities that provide these patients with an additional supportive relationship. The presence of certain diagnoses, such as sepsis, may actually serve as a marker for hospitalized patients who have other underlying health care concerns, and their follow-up care once discharged may need to be further individualized. For instance, a medication review and education on use may need to be provided to patients with a large number of prescriptions. In addition, these patients may benefit from more frequent clinic or home visits.

Finally, the patients in the HfR group had about an $80 \%$ turnover rate, indicating the list of these specific patients is fairly dynamic. As such, interventions to decrease the number of patients frequently readmitted to decrease global readmission rates should consider addressing patients with certain characteristics (eg, age, CCI, MFS, and medication and problem lists) rather than focusing on specific patients. Furthermore, the ability to predict patients who may become HfR members is probably more important than understanding the specific individuals if the goal is to reduce readmissions.

Several limitations of this study are present. The study included patient care data obtained retrospectively from a large academic health center hospital located in a southeastern US city. Therefore, the results and conclusions may not be applicable to other hospitals of varying types, size, or locations. In addition, the data utilized in this study were obtained from the hospital's administrative informational system, and a mechanism to confirm accuracy was not available. Finally, readmitted patients were classified into the groups based on the number of readmissions in a calendar year; it is possible that the overall number of patients classified into the HfR group would have been higher if a rolling readmission rate was utilized. Of the 30 patients who experienced readmissions separated by 2 consecutive calendar years, 28 of these patients were already classified in the HfR group. Therefore, using this alternative method would have little if any impact on our results.

In conclusion, the group of patients who experience 3 or more readmissions to the hospital in a calendar is associated with several characteristics: younger age; single; have psychiatric, substance abuse, or chronic pain diagnosis; and have longer medication and problem lists. A further evaluation of these characteristics may provide additional information and allow the ability to intervene earlier with patients before the readmission. Finally, the list of specific individual patients considered to be high users for hospital readmissions was dynamic and changed during 3 consecutive years, and addressing the needs of these specific patients may not be an appropriate strategy to reduce global readmission rates.

To see this article online, please go to: http://jabfm.org/content/ 32/1/58.full.

\section{References}

1. Jencks SF, Williams MV, Coleman EA. Rehospitalization among patients in the Medicare fee-for-service program. N Eng J Med 2009;360:1418-28.

2. Berenson RA, Paulus RA, Kalman NS. Medicare's readmission-reduction program-a positive alternative. N Engl J Med 2012;366:1364-6.

3. Krumholz HM, Wang K, Lin Z, et al. Hospitalreadmission risk-isolating hospital effects from patient effects. N Eng J Med 2017;377:1055-64.

4. Health policy brief: Medicare hospital readmissions reduction program. Health Affairs. Available from: http://healthaffairs.org/healthpolicybriefs/brief pdfs/healthpolicybrief_102.pdf. Published November 12, 2013. Accessed June 22, 2014.

5. Joynt KE, Jha AK. A path forward on Medicare readmissions. N Engl J Med 2013;368:1175-7.

6. Coleman EA, Parry C, Chalmers S, Min SJ. The care transitions intervention: results of a randomized controlled trial. Arch Intern Med 2006;166:1822-8.

7. Jack BW, Chetty VK, Anthony D, et al. A reengineered hospital discharge program to decrease rehospitalization: a randomized trial. Ann Intern Med 2009;150:178-87.

8. Naylor MD, Brooten D. Campbell R, et al. Comprehensive discharge planning and home follow-up of hospitalized elders: a randomized clinical trial. JAMA 1999;281:613-20.

9. Hansen LO, Young RS, Hinami K, Leung A, Williams MV. Interventions to reduce 30-day rehospitalization: a systemic review. Ann Intern Med 2011; 155:520-8.

10. Leppin AL, Gionfriddo MR, Kessler M, et al. Preventing 30-day hospital readmissions: a systemic review and meta-analysis of randomized trials. JAMA Intern Med 2014;174:1095-107.

11. Garcia-Perez L, Linvertova R, Lorenzo-Riera A, Vazquez-Diaz JR, Duque-Gonzalez B, Sarria-Santamera A. Risk factors for hospital readmissions in elderly patients: a systemic review. Q J Med 2011; 104:639-51. 
12. Calvillo-King L, Arnold D, Eubank KJ, et al. Impact of social factors on risk of readmission or mortality in pneumonia and heart failure: systemic review. J Gen Intern Med 2012;28:269-82.

13. Garrison GM, Mansukhani MP, Bohn B. Predictors of thirty-day readmission among hospitalized family medicine patients. J Am Board Fam Med 2013;26: 71-7.

14. Regenstein M, Andres E. Reducing hospital readmissions among Medicaid patients: a review of the literature. Q Manage Health Care 2014;23:20-42.

15. Logue E, Smucker W, Regan C. Admission data predict high hospital readmission risk. J Am Board Fam Med. 2016;29:50-9.

16. Barnett ML, Hssu J, McWilliams JM. Patient characteristics and differences in hospital readmission rates. JAMA Inter Med 2015;175:1803-12.
17. Zook CJ, Moore FD. High-cost users of medical care. N Engl J Med 1980;302:996-1002.

18. Schrag D, Xu R, Hanger M, Elkin E, Bickell N, Bach P. Fragmentation of care for frequently hospitalized urban residents. Med Care 2006;44:560-7.

19. Raven MC, Billings JC. Goldfrank LR, Manheimer ED, Gourevitch MN. Medicaid patients at high risk for frequent hospital admissions: real-time identification and remediable risks. J Urban Health 2009; $86: 230-41$.

20. Ranasinghe I, Wang Y, Dharmarajan K, Hsieh AF, Bernheim SM, Krumholz HM. Readmissions after hospitalization for heart failure, acute myocardial infarction, or pneumonia among young and middleaged adults: a retrospective observational cohort study. PLoS Med 2014;11:e1001737. 